\title{
NATURALEZA SINTÁCTICA DE LAS FORMAS SIGLARES. EL CAMBIO FUNCIONAL
}

\author{
FÉLIX RODRÍGUEZ GONZÁLEZ \\ (Universidad de Alicante)
}

0. La acuñación y empleo de las siglas en la época actual recibe un impulso de la necesidad de reducir de algún modo la continua referencia a expresiones de extremada longitud que tienen lugar en las áreas de la ciencia, la administración y la política, principalmente, como resultado de factores tales como el progreso científico-técnico y la proliferación de partidos políticos. La referencia a sectores científicos, partidos y organizaciones de diversas clases, fuente principal del ingente y creciente caudal de siglas, explica el carácter sustantivo de éstas ${ }^{1}$. Ocasionalmente sin embargo, la sigla pertenece a otras categorías gramaticales. El objetivo de este estudio es, precisamente, apuntar la variedad de roles sintácticos desempeñados por las siglas en español e inglés, no sólo los derivados de su forma original sino también los adquiridos por efecto del cambio funcional y las transformaciones semánticas que pueden sufrir en el curso del tiempo. Asimismo intentaré definir el status lingüístico, la relativa frecuencia así como los contextos en que aparecen los diversos tipos.

1. La mayoría de las siglas, en virtud de la naturaleza predominantemente político-social de las denominaciones, y por tanto su carácter sustantivo, viene determinada por el nombre central de la frase subyacente ( $P=$ Partido en $P S O E)$ o bien por un concepto asociado ('servicio secreto' en FBI), que se hace más necesario cuando el término prinipal no está representado en el lexema siglar (el ANZUS 'Australia, New Zealand and United States) ${ }^{2}$.

1 Las siglas recogidas en los diccionarios testimonian este hecho, también reflejado en algunas definiciones del propio término, como ésta que recojo de un periódico argentino dentro de una nota titulada "Instituciones: Las siglas, un idioma aparte»: “Se llama sigla a la abreviatura formada por iniciales o sílabas que identifica a entidades públicas o privadas, sociedades comerciales, deportivas, culturales, etcétera".

2 Para un examen detallado de la frecuencia y complejidad de tales asociaciones, asi como de sus repercusiones morfológicas a la hora de determinar el género de las siglas, véase Rodríguez González (1983b; 1984a). 
Por asociaciones semejantes el valor sustantivo se superpone incluso a siglas cuyas frases subyacentes no son de tipo nominal sino verbal. Tal es el caso de la entidad VIA (Valorizar la Innovación en el Amoblamiento) ${ }^{3}$ y el colectivo militar portugués conocido con el lema SUV (Soldados Unidos Vencerán) ${ }^{4}$; más excepcional resultará aún la Asociación ACECA (Cantabria es Castilla) ${ }^{5}$ cuya estructura oracional permite definir expresamente la posición política de este grupo político regional. En todas ellas puede observarse una función apelativa ligada al mensaje político o publicitario que intentan transmitir, lo cual contrasta con los constituyentes nominales puramente descriptivos que normalmente subyacen a las denominaciones sigladas.

Hasta aquí la referencia a la sigla como denominación de entidades o instituciones. En menor número se siglan también designaciones de nombres muy concretos referidos a personas (PNN, ATS) u objetos (TV, OVNI), si bien lo más frecuente es que éstos se obtengan por metonimia de las instituciones que les dieron origen; así llamamos un PAS al empleado que forma parte del colectivo PAS (Personal de Administración y Servicios) y un SEAT al coche de la SEAT (Sociedad Española de Automóviles de Turismo) ${ }^{6}$.

Un carácter menos concreto es el que caracteriza a un tipo de siglas cada día más frecuente, que merece destacarse por su novedad ya que ha hecho irrupción en los últimos años. Se trata de largos enunciados constituidos por nombres comunes referidos a conceptos en uso en los lenguajes técnicos especializados de la economia, la administración, la ciencia, etc.; e.g.: ACM (acumulación mundial del capital) $^{7}$, EOS (estimación objetiva singular), ILT (incapacidad laboral transitoria) ${ }^{8}$, MAP (máximo accidente que pueda suponerse) ${ }^{9}$, OPA (oferta pública de compra) ${ }^{10}$, TBR (tasa bruta de reproducción) ${ }^{11}$, TPI (tasa de paro insoportable) ${ }^{12}$, TRBC (toneladas anuales de registro bruto compensado) ${ }^{13}$. Como puede observarse se trata de meras «expresiones fraseológicas» cuya siglación hubiera llamado la atención en épocas pasadas pero que, con el tiempo, por cumplir la doble condición de prolijidad y uso frecuente _en los dominios que les son propios-, han acabado siendo objeto de abreviación. La prensa periódica constituye un fértil campo de cultivo al darles fácil acogida en sus secciones especializadas. A primera vista parece como si se tratara de formaciones efímeras de las que el periodista o

3 Cambio $16,16-1-84$, p. 87.

4 Cambio 16, 13-10-75, p. 48.

5 Revista de Estudios Politicos, 19 (1981), p. 160.

6 Si destaco su frecuencia relativa en uno y otro caso es en atención a su uso en la lengua general, haciendo caso omiso de los lenguajes técnicos de la ciencia, la medicina, electrónica, etc., donde cabe esperar una abundancia de nombres concretos.

7 Triunfo, marzo 1982, p. 7.

8 El Pais, 2-10-82, p. 19.

9 «En los niveles MAP (máximo accidente que pueda suponerse) o super MAP» (Cambio 16, 2-8-76, p. 28).

10 Asi aparece en $S P, 2-2-69$, p. 47, probablemente tomada del francés $(A=a c h a t$ 'compra'). Martínez de Sousa la registra como Oferta pública de adquisición (1984).

11 Cambio 16, 24-4-72, p. 32.

12 El Pais, 16-8-84, p. 31.

13 El Alcázar, 14-9-84, p. 7. 
escritor se sirve en un determinado momento para ahorrar espacio en un artículo o reportaje donde se mencionan varias veces. Pero esto que puede ser cierto en su origen, al menos en algunos casos, carece de relevancia cuando se comprueba su recurrencia en diferentes textos y la estereotipación de que gozan sus lexemas siglares. Aún más, posteriormente algunas de ellas reciben el espaldarazo definitivo al saltar al medio televisivo: citaré por ejemplo IPC (indice de precios al consumo) y ZUR (zona de urgente industrialización) que he oído repetidas veces en los noticiarios; téngase en cuenta que el lenguaje de la televisión es básicamente un lenguaje escrito, en especial el de estos comunicados, que se escriben para ser leídos (cf. Seco 1977: 187).

2. Como he venido apuntando, las siglas tienen esencialmente una función sustantiva, cualquiera que sea la variedad examinada. Ahora bien, aunque en número mucho más reducido, cabe considerar asimismo aquéllas que en razón de la base o del valor del sintagma pertenecen a otras categorías gramaticales. A este respecto el inglés ofrece una casuística muy variada: verbos (to T.O. 'to turn over one customer to another'; to O.C. 'to overcharge deliberately) ${ }^{14}$, adjetivos (P.D. 'plain drunk' ${ }^{15}$; D.O.A. 'dead on arrival' ${ }^{16}$ ), adverbios (I.V. 'intravenously' 17; p.m. 'post meridiem' y a.m. 'ante meridiem'), interjecciones (S.O.B. 'son of a bitch') e incluso frases de variado tipo (i.a. 'if awake' ${ }^{18}$; c.f. fr. SVP 's'il vous plaît').

Este tipo de siglas no nominales tienen por lo general un carácter jergal, pertenecen al slang particular de un oficio o profesión (médicos, comerciantes, etc.) ${ }^{19}$; excepción hecha de algunas siglas, como p.m., a.m. (o PM, AM), de amplio uso y abreviaturas en su origen, o S.O.S., expresión eufemística de la lengua general. También puede ocurrir que una de estas siglas forme parte del lenguaje especial de un grupo restringido y después pase a la lengua común; es el caso de snafu (situation normal all fouled up), adjetivo 'confuso, complicadísimo', proveniente del argot militar y cuya popularidad dio lugar en su día a una larga serie de siglas con las inciales FU, entre ellas fubar (fouled up beyond all recognition), fubb (fouled up beyond belief), sapfu (surpassing all previous foul-ups) ${ }^{20}$.

El uso de tales siglas no constituye un desarrollo reciente del inglés americano

14 T. O. y O. C. forman parte del léxico cie los vendedores de zapatos (American Speech, 12, 2(1938), p. 150). De la guerra del Vietnam es WHAM, empleada como acortamiento de "Win hearts and minds" (Time, 6-11-72, p. 23).

15 Mencken (1945: 412).

16 Del argot de la medicina (Amer. Speech, 36, 2 (1961), p. 145).

17 lbid., p. 145.

18 Barkley (1927: 313).

19 Igualmente del argot de la enfermería del inglés americano son las siguientes expresiones (Kolin 1980): AMA (against medical advice), PTA (prior to admission), TBA (to be admitted), TKVO (to keep vein open), TNTC (too numerous to count), SOAP (subjective, objective, assessment, plan) empleado con valor verbal (eg., to soap a patient) 'trazar sobre un diagrama la asistencia de un paciente'; del slang del ejército ("Glossary of army slang", Am. Speech, 16, 3 (1941), 163-69): IC (inspected and condemned), O.A.O. (one and only), e incluso una oración completa, R.H.I.P. (rank has its privileges); de la jerga de los vagabundos (Klein 1926): C.H. and H. (cold, hungry and dry), L.S. and M.S. (less sleep, more spedd).

20 Am. Speech, 30, 3 (1945), p. 226. Cf. También Baum (1955: 103, 104). 
sino que, al contrario, su presencia se detecta en distintas épocas, incluso en las más tempranas; asi, al primer brote de siglas que se conoce en el uso que hoy damos al término-, hacia 1839, pertenecen O.K. ('all correct'), D.K. (don't know), N.G. (no good) ${ }^{21}$. A principios de siglo se documentan un buen número de ellas en la recopilación llevada a cabo por Long (1913) entre las que figuran c.y.k. (consider yourself kised), t.h.b. (family hold back), m.i.k. (more in the kitchen), etc., las cuales llaman la atención por el motivo entre lúdico y criptográfico que presidió su acuñación.

La relativa frecuencia de este tipo de siglas tampoco me ha pasado desapercibida en indonesio, una lengua donde las siglas se incorporan al sistema lingüístico en una proporción tal que deja perplejos a cuantos examinan este aspecto del léxico (cf. Makkai 1974: 361; De Vries 1970: 344). Efectivamente, a esta lengua pertenecen frases 'acronimizadas' 22 del tipo berdikari (berdiri diatas kaki sendiri 'mantenerse sobre sus propios pies'), acuñada por el ex-presidente Sukarno; pero lo que más me sorprende es hallar abreviada en forma de sigla (L.M.M.) una simple oración de la lengua ordinaria (Langit Makin Mendung 'el cielo se está nublando'), algo tampoco desconocido en el sundanés, una de las lenguas regionales, que cuenta con abreviaciones como blagtjingpo (panto ngadjeblag utjing nempo 'si abres la puerta verás el gato'=está claro como la luz del día) ${ }^{23}$.

Por su parte el español, al igual que otras muchas lenguas, casi desconoce este tipo de siglas no nominales. Entre las pocas existentes se encuentran algunas tan antiguas como U.H.P. (Uníos, Hermanos Proletarios), o CAFE (Camaradas, Arriba Falange Española) y VERDE (Viva El Rey De España) cuyos lemas o consignas recurrieron a la sigla como instrumento mnemónico (cf. Rodríguez González 1983a: 211); del léxico de hoy podrían citarse igualmente NP (no presentado, adj.), oída en los claustros de evaluación de los profesores de enseñanza secundaria y, entre los estudiantes mayormente, la expresión n.p.i. (ni puta idea) ${ }^{24}$, que al igual que el

21 Cit. por Heflin (1941).

22 Un detenido estudio de la terminología de las abreviaciones y siglas puede verse en Martinez de Sousa (1984: 15-65) y Rodriguez González (1984b).

23 Cit. por De Vries (1970). El espectro sintáctico de las siglas en indonesio es de tal amplitud que se dan casos, supuestamente inéditos en muchas otras lenguas, donde cada grafema representa un concepto bastante complejo formado a partir de frases u oraciones. Esto ocurre por ejemplo con algunos programas politicos que adoptan la forma siglar como medio de conseguir una más fácil evocación. Así, la sigla USDEK (cf. Dardjowidjojo 1974: 148) representa:

U = Undang2 Dasar 1945 'la Constitución de 1945'

$\mathrm{S}=$ Socialisme Indonesia 'Socialismo indonesio'

D = Demokrasi Terpimpin 'Democracia dirigida'

$\mathrm{E}=$ Ekonomi Terpimpin 'Economía dirigida'

$\mathrm{K}=$ Kepribadian Indonesia 'Identidad indonesia'

Del mismo modo Nekad ('decididos sin temor', cf. Vries 1970) significa:

$\mathrm{N}=$ Negara kita persatukan 'nosotros unificamos nuestro país'

$\mathrm{E}=$ Ekonomi kita sosialiskan 'nosotros socializamos nuestra economía

$\mathrm{K} \times$ Keamanan kita se/enggarakan 'nosotros mantenemos la seguridad'

A = Agama kita muliakan 'nosotros glorificamos la religión'

D = Demokrasi terpimpin kita adjalankan 'nosotros practicamos una democracia dirigida'

24 La iuerza de arrastre del paradigma nominal es tal que incluso una frase como n.p.i., o NPI, puede verse despojada de su verdadero valor y asumir una función sustantiva, como se pone de manifiesto en el siguiente texto: 
inglés S.O.B. o el indonesio I.D.H. (indehoj 'hacer el amor') ha sido motivada eufemisticamente ${ }^{25}$.

3. El escaso número de siglas no nominales se ve relativamente incrementado por medio del cambio funcional; es decir, siglas que por su base son sustantivas "cambian" de categoría adoptando funciones adjetivas, verbales, etc. Un cambio muy frecuente en las siglas es el de nombre a adjetivo en función atributiva; e.g.: españoles no DC, nombres UCD, neutralidad TV, nueva violencia ETA, conductores $E M T$, etc.; construcciones similares se repiten sin cesar también en otras lenguas: fr. émissions $t v$, it. televisione USA, etc.

La doble función de sustantivo y adjetivo homólogo en un mismo lexema se produce en sintagmas cuya estructura profunda cuenta con nexos prepositivos: hombres (de la) UCD, violencia (de la) ETA, etc. Al elidirse éstos los elementos resultantes quedan yuxtapuestos, resultado que es favorecido por expresiones en aposición muy frecuentes en la lengua y estructuralmente muy similares: el partido $U C D$, la organización ETA, etc. En este segundo grupo de ejemplos la sigla mantiene una relación copulativa con el segundo elemento del sintagma, es decir, se trata del partido que es o se llama UCD, y de la organización que se llama ETA. La yuxtaposición entre ambos sustantivos es total por cuanto presentan una unidad funcional; incluso en casos como éstos forzando un poco la sintaxis se podría admitir la preposición de: el partido de la UCD, la organización de ETA. Tal convergencia de estructuras por un lado, y por otro la naturalidad de la construcción apositiva, contribuyen a la irradiación de este segundo esquema. De este modo la construcción originariamente determinativa, desprovista ahora del nexo prepositivo y flexión gramatical - de género y número- se revela como una expresión muy económica, pronta a ser aprovechada en la lengua, sobre todo escrita, y de un modo especial en los titulares.

La polivalencia funcional de la iglesia es aplicable a un extenso número de lexemas, cada vez más variado, de suerte que es difícil pensar en una sigla que se resista a estos cambios. En opinión de algunos autores (Pettenati 1955; Leso 1978: 17), sin embargo, la sigla PC (Partido Comunista), o bien PCl (Partido Comunista Italiano), en italiano se limita al uso sustantivo. Probablemente se haya pensado, desde la perspectiva de imagen visual, en el obstáculo que supone el grafema $P$

« ¿..si eso es así, para qué los cursillos y especialidades, para mandar a un licenciado en filología inglesa a un parvulario o un señor maestro $\sin N P I$ del asunto a una unidad de educación especial? ("Correo del mutualista", Muface, nov. 1984, p. 5.)

Por anómala que parezca esta construcción no hay que olvidar, por un lado, las restricciones sintagmáticas impuestas por el carácter estereotipado del lexema siglar, que impide se efectúen transformaciones del tipo $\sin ^{\star} P I$ o $n^{\star} P I$, como correspondería a sus expresiones completas (sin puta idea, ni puta idea); y por otro, la menor conciencia que se tiene de los elementos que constituyen cualquier enunciado por el mero hecho de someterse a abreviación.

25 Igualmente entre amigos chilenos he oído la expresión ele jota(eme)>L.J.(M) basada en la pronunciación dialectal de nos fuimos (mierda)/loh xuimoh/ y utilizada para significar 'vámonos'.

Fiel indicativo del móvil eufemístico que condujo a estas siglas, así como a otras similares del inglés (N.S. 'no shit', T.S. 'tough shit', etc.), es la escasa o nula reducción del cuerpo fonético pues, como se observará, el número de sílabas se mantiene prácticamente invariable. 
antepuesto para la asignación del significado 'comunista', más susceptible de ser evocado por la $C$, en contraste con la homografía de las iniciales en siglas como DC (Democracia Cristiana, democrático cristiano), t.b.c. (tuberulosi, tuberculótico), TV (televisione, televisivo), etc., las cuales por su estructura se muestran más propensas a la bifuncionalidad.

Ahora bien, tales discrepancias no constituyen hoy impedimento alguno como lo atestigua la misma $\mathrm{PCl}$ en un texto recogido de la prensa italiana reciente: «Dirigente pci affianca il sindico Valenzi per imprimire a Napoli un nuevo corso"; idéntico cambio funcional he encontrado también en español: "Berlinguer aparece seguido del PCl, Eduardo Perna, jefe del sector comunista del Parlamento...». Lo mismo puede decirse de las diversas siglas que comparten los grafemas iniciales PS (Partido Socialista), como se pone de manifiesto en la siguiente cita: «El gabinete Mario Soares, frágil al no contar con mayoría parlamentaria, tiene un marcado cariz $P S$...". No conviene olvidar, por otra parte, que la función modificante de las siglas $\mathrm{PCl}$ y PS, u otras similares como las españolas PCE o PSOE, se ve favorecida por condicionamientos de orden extralingüístico ya que su empleo proporciona al texto una precisión referencial de la que carecen los calificativos comunista y socialista, etiquetas comunes, como se sabe, a múltiples denominaciones de partidos y grupos políticos de ideología afín (cf. Rodríguez González, 1980: 150 ss.).

La plasticidad adquirida por las siglas no se limita al valor adjetival más normal, en grado positivo, pues en ocasiones son objeto también de ponderaciones de tipo comparativo y superlativo: "pequeño pero tan IBM como los grandes" 38; "Garrigues Walker (...) es muy USA» ${ }^{39}$; «Es un libro muy CC ${ }^{40}$; cf. en francés «un ton très $S A C \gg 41$.

Aunque con menor frecuencia el cambio funcional también se produce hacia otras categorias como el verbo; en español, por ejemplo, de MATESA se ha formado matesar, de CNT cenetear, de PSOE psoerse, como en "no te psoes, Boyer» (cf. Rodríguez González, 1980: 172 ss.) ${ }^{42}$.

En el cambio de una categoría (nombre) a otra (adjetivo, verbo) en español los dos patrones apuntados difieren en cuanto a su estructura. En efecto, mientras en el primero la base se mantiene inalterable, en el segundo adquiere los sufijos flexivos propios de la categoría verbal. Por otra parte la motivación que preside estas formaciones es también distinta: el cambio de nombre a adjetivo responde a impulsos económico-expresivos, el de nombre a verbo constituye más bien un recurso retórico en el que el neologismo adquiere un fuerte poder llamativo. Ligado a estos fines está el contexto en que aparecen; así, mientras las primeras construcciones son muy comunes, y por ende, muy propicias a emplearse en cualquier momento y por

\footnotetext{
38 Cambio 16, 16-6-75, p. 25 (anuncio).

39 Francisco Umbral, «Spleen de Madrid", El País, 17-3-83, p. 35.

40 Titular tras el que se lee: «...es un libro muy $C C$ (...) porque coincidentemente aúna las dos grandes ideas - Catalunya y Cristianismo- que lo presidieron» (Destino, 16-11-78, p. 21).

41 Le Canard Enchaîné, 29-6-77, p. 3. SAC=Service d'action civique.

42 Del mismo modo cabría considerar el cambio que puede operar dentro de una misma categoría, como el que tiene lugar dentro del nombre. Ya vimos al principio, por ejemplo, el producido en la SEAT $\rightarrow$ el Seat, esto es del nombre propio al nombre común; asimismo, podría citarse la (sociedad) IBM $\rightarrow$ la (computadora) IBM o ibeeme, entre otros.
} 
un gran número de usuarios, las segundas tienen un marco mucho más limitado como es el del ámbito político o humorístico.

En inglés las formaciones verbales a partir de nombres son más frecuentes: $M C$ (Master of ceremonies) $\rightarrow$ to $M C$ (to emcee) ${ }^{43}$, $\mathrm{KO}$ (knock out) $\rightarrow$ to $\mathrm{KO}^{44}, \mathrm{RV}$ (Recreational vehicle) $\rightarrow$ to RV (to travel in a RV) ${ }^{45}$, TV (television) $\rightarrow$ to $T^{46}$, etc. También cabe registrar el fenómeno opuesto, esto es, una sigla que toma un valor de sustantivo o nombre que no le corresponde por su forma originaria, y esto no sólo a partir del verbo (to H.O. 'hold money' $\rightarrow$ H.O. 'money so withheld') ${ }^{47}$ sino también a partir de frases adjetivales, como a NIP (not in possession) 'una persona que no lleva consigo el carnet de conducir' ${ }^{48}$, a kia (killed in action) 'soldado muerto en acción' ${ }^{49}$. Pero lo que más llama la atención de esta lengua es la flexibilidad funcional de las siglas, o sea, la pronta disposición a asumir múltiples funciones gramaticales como si de cambios simultáneos y automáticos se tratara. La sigla OK, por ejemplo, sin duda la más frecuente de todas en la conversación corriente, adopta los valores más variados: a su valor básico de interjección (¡está bien!, ¡vale!) añádese el de adjetivo 'correcto, conforme' (it's OK with me), adverbio 'satisfactoriamente' (I did it OK), verbo 'aprobar', 'dar el visto bueno a' (House OKs death penalty) ${ }^{50}$, nombre 'aprobación', 'visto bueno' (I gave him the OK) ${ }^{51}$

Esta multiplicidad de funciones puede darse con muchas otras siglas, como las que cito a continuación recogidas del argot de los hospitales americanos (Barkley 1927): La sigla p.m., por ejemplo, originariamente adverbial (v.g., 5 p.m. 'de la tarde') se emplea también con valor nominal; así, de una enfermera que está de permiso

43 «Leon will emcee for the occasion", Hollywood Citizen, 14-9-37 (cit. en Am. Speech, 15, 3 (1940), p. 328.

44 Expresión ampliamente difundida en la jerga boxística ('poner fuera de combate', 'dejar K.O.', 'noquear'), de donde se ha extendido a la lengua general que lo emplea en un sentido figurado; e.g.: "Watergate movie should KO Rocky's honors bid" (Edmonton Jornal, 26-3-77, p. 37); "Coliseum patron's seat KO'd at boxing match» (Edmonton Journal, 1-4-78, B13). Este uso adjetivado ha pasado al español con la misma forma que el nombre K.O. (que el pueblo pronuncia /káo/: «Me quedé cao»); cf. Seco (1977: 194).

45 "...it costs them less to go RVing", Family Weekly, 16-4-78, 24/2; cit. en Am. Speech, 56, 3 (1981), p. 218.

46 «... council is TV-ed evicting tenants», Spectator, 5-9-70. Tan común es la flexión -ed del participio que se documenta incluso precediendo a otra sigla dentro de un compuesto formado por dos lexemas siglares: "more and more MIRVed ICBMs", "their MIRVed ICBMs"; en el mismo texto aparece explicitado su significado ( «ICBMs with multiple warheads or MIRCs") con el que mantiene una misma línea de correferencia.

47 R. Oliver, «More Carnie Talk from the West Coast», Am. Speech, 41 (1966), p. 281.

48 "l thought he had no licence, but it turned out that he was just a NOP» (D. Dempsey, «the Language of Traffic Policemen», Am. Speech, 37, 1962, pp. 266).

49 “He was a 'kia'», New York Times", 28-4-44, p. 7. Cf. también Baum (1955: 107).

50 Chicago Tribune, 3-12-76, p. 1, sec. 1; «...please have your cheques stamped and $O K K^{\prime}$ for purchases at office before you start shopping», rezaba un letrero del supermercado «Safeway» de Edmonton en 1978. El estilo «telegráfico» (cf. nota 33) lleva incluso a prescindir del apóstrofo, como en los dos titulares siguientes: "Racial quotas OKd in reapportionment" (Pittsburgh Courier, 12-3-77, p. 1); "Rigorous New Ethics Code $O K d$ by House, 402 to 22" (Los Angeles Times, 3-3-77, p. 1). Otras veces el término aparece completamente lexicalizado: «AIB okays gasoline price jump» (Edmonton Journal, 26-2-77, p. 1).

51 En este mismo valor se recoge en español, lo que da idea de la popularidad del préstamo: «...es un decidido partidario de llegar a un acuerdo parlamentario de seis meses de duración con los peronistas para que éstos obtengan de los sindicatos el “OK» a un pacto que fijaría precios y salarios..." (Diario 16, 3-11-83, p. 10). 
"de tarde» se dice que está “on her p.m.» ${ }^{52}$, o en forma verbal, “is p.m.ing". El alimento suplementario del niño se convierte en su "two or six o'clock p.c.", de la frase latina post cibum; como verbo se emplea en expresiones como «i had six babies to p.c. tonight», pero también un medicamento puede darse «p.c.» o «a.c.» (ante cibum), frases en las que asume valor adverbial. Si un médico desea que se administren aspirinas al enfermo cuando sea necesario pedirá «Aspirin grane five p.r.n.", y esta frase abreviada del latín pro re nata a veces se torna adjetivo, "a p.r.n order», o verbo, «Did he p.r.n. that medication?», y también, por supuesto, nombre, "You can have a p.r.n., then you won't have to call me».

Expresiones como estas son susceptibles de emplearse en otros argots profesionales siempre que la sigla adquiera un alto índice de presencia. Entre los estudiantes graduados de las universidades americanas y canadienses, por ejemplo, donde la expresión «(Graduate) Teaching Assistantship» es de uso muy común, se emplea no sólo la forma T.A. sino también frases como «l'm a T.A." (=teaching assistant) 'soy profesor ayudante', "l'm going to T.A.» (=teach) 'voy a dar clase (en calidad de profesor ayudante)', etc.

En inglés el fenómeno del cambio funcional o «metábasis», mucho más arraigado que en español y otras lenguas románicas, ha recibido tradicionalmente el nombre de conversión. El caudal de neologismos que entra en la lengua inglesa por este procedimiento supone el $2 \%$, aunque muchos más se utilizan como palabras efímeras para conseguir efectos estilísticos (Simonini 1966: 755 ) ${ }^{53}$. Tan extenso es el fenómeno en inglés que se suele hacer de él un estudio aparte, separándolo de la derivación, su congénere más natural. Ambos tienen de común la «transcategorización», es decir, el cambio o conversión de una categoría a otra. El principio de clasificación, sin embargo, se basa en la naturaleza de los morfemas sufijales, o sea, en la distinción entre sufijos flexivos (en el verbo: $-s,-i n g,-e d$ ) y derivativos. La "conversión» engloba todas las palabras que quedan 'convertidas' en otras categorías, sin cambiar el lexema, o bien adoptando únicamente las flexiones propias de su categoría, es decir, sufijos flexivos pero nunca derivativos.

Algunos gramáticos como Zandvoort (1972: 265) encuadran la "conversión» fuera de la "formación de palabras" (word formation) conceptuándola más como un proceso sintáctico que derivativo. Otros como Quirk et al. (1972: 1.009), en base a la función transcategorizante, inherente también a la derivación, y, por otro lado, considerando que la ausencia de sufijo constituye en definitiva un sufijo $\varnothing$, sitúan la conversión dentro de la formación de palabras, como un tipo especial de derivación al que se le da el nombre también de «derivación $\varnothing$ »54. De esta forma se pone de relieve el entrecruzamiento de la sintaxis y la formación de palabras. Al objeto de distinguir ambos niveles, en particular sus reglas de formación, Quirk (p. 976), por

52 Cf. también: «Public School boy, 18, has free p.m.s. (=afternoons), wishes to make pocket money" (anuncio; cit. por Müller-Schotte (1968: 110).

53 La facilidad que tiene el inglés para cambiar de una categoria a otra, práctica que alcanzó su punto más álgido en tiempos de Shakesperare, se explica por la pérdida de flexiones que esta lengua ha sufrido a lo largo de su historia (cf. Williams 1975: 134).

54 Cf. también Adams (1973: cap. IV). El calificativo $\varnothing$ (zero) ha tenido una larga tradición en la lingüistica estructural americana donde el fenómeno ha recibido los nombes de «zero-elements» y "zeroalternants» (Bloomfield), "zero modification» (Bloch y Trager), «zero change» (Narváez 1959). 
su parte, considera el factor productividad como criterio que permitiria enmarcar un determinado cambio funcional en un plano sincrónico (como un proceso sintáctico) o diacrónico (como un proceso léxico-formativo). De este modo puede pensarse que la flexibilidad de una sigla nominal, como UCD, al convertirse en adjetivo (hombres $U C D$ ) está más cerca del nivel sintático, mientras que el segundo grupo de formaciones verbales (matesar, cenetear, etc.), a tenor del menor índice de frecuencia y de su caráter novedoso, pueden considerarse más bien en una perspectiva histórica, es decir, como verdaderas creaciones léxicas. Por este motivo volveré a ocuparme de ellas más extensamente al tratar de la formación de palabras a partir de las siglas.

\section{Referencias bibliográficas}

Adams, Valerie (1973), An Introduction to Modern English Word-formation, London, Longman.

Barkley, Dorothy (1927), "Hospital Talk», American Speech, n. ${ }^{\circ} 7$, pp. 312-14.

Baum, S. V. (1955), «From 'AWOL' to 'VEEP': the Growth and Specialization of the Acronym", American Speech, 30, pp. 103-110.

Caso, Arthur Lewis (1980), "The Production of New Scientific Words", American Speech, 55, pp. 101-11.

Dardjowidjojo, Soenjono (1979), "Acronymic Patterns in Indonesian», South-East Asian Linguistic Studies, 3, pp. 143-160.

Heflin, W. A. (1941), «O.K. But what do we know about it?", American Speech, 16, n. ${ }^{\circ}$ 2.

Kolin, Philip C. (1980), «More Nursing Terms", American Speech, 55, 1, pp. 46-53. Leso, Erasmo (1978), "Vocabolari recenti di lingua politica», Lingua Nostra, 39, 1, pp. 11-19.

Long, Percy W. (1915), «Semi-secret Abbreviations», Dialect Notes, 4, 3, pp. 245-46. Makkai, Adam (1974), "Acronymy in English: A Stratificational Re-examination», Proceedings of the Eleventh International Congress of Linguistics, ed. Luigi Heilmann, Bologna: il Mulino, pp. 345-63.

Martínez de Sousa, José (1984), Diccionario internacional de siglas y acrónimos, 2. ${ }^{a}$ ed., Madrid, Pirámide.

Mencken, H. L. (1945), The American Language, Supplement I, New York, Knopf. Müller-Schotte, Hans (1968), "Zur Theorie und Praxis der englischen Abkürzungen», Lebende Sprachen, 13, N. ${ }^{\circ} 4-5$, pp. 105-12.

Narváez, R. A. (1959), A descriptive analysis of Word-Formation in Old Spanish (tesis doctoral inédita), Univ. of Minnessota.

Pettenati, G. (1955), “A proposito di sigle», Lingua Nostra, 16, 3, p. 91.

Pyles, Thomas y Algeo, John (1982), The Origins and Development of the English Language, 3. ${ }^{\mathrm{a}}$ ed., New York, Harcourt Brace Jovanovich.

Quirk, Randolph et al. (1972), A Grammar of Contemporary English. London, Longman.

Rodríguez González, Félix (1980), Estudio lingüistico de las siglas en español actual (tesis doctoral inédita), The University of Alberta. 
- (1983a), "On the Coining of Acronyms by Homonymy", Anglo-American Studies, 3, 2, pp. 209-221.

- (1983b), «Problemas planteados en la asignación del género de siglas extranjeras", Actas del / Congreso Nacional de Lingüística Aplicada (AESLA), Murcia, pp. 277-86.

- (1984a), "El género de las siglas», Revista Española de Lingüística, 14, 2, pp. 311-65.

- (1984b), «Taxonomia de la siglación», Proceedings of the 7th World Congress of Applied Linguistics (extracto), ed. J. Den Haese y J. Nivette, vol. 3, Bruselas: ITO/VUB, p. 1.221. Versión ampliada en preparación.

Seco, Manuel (1977), "El léxico de hoy», en R. Lapesa (coord.), Comunicación y lenguaje, Madrid, Karpos, pp. 183-201.

Simonini, R. C. (1966), «Word-Making in Present-Day English», English Journal, 55, pp. $752-57$.

Vries, J. W., de (1970), "Indonesian Abbreviations and Acronyms", Bijdragen tot de Taal-Land-en Volkenkunde (Holanda), 126, pp. 338-46.

Williams, Joseph M. (1975), Origins of the English Language, New York, Free Press. Zanvoort, R. W. (1972), A Handbook of English Grammar, 6. ${ }^{a}$ ed., London, ongman. 